\title{
Pancreatic Neuroendocrine Neoplasm Associated with a Familial MAX Deletion
}

Authors

Sandrine Petignot ${ }^{1}$, Adrian F. Daly ${ }^{1}$ D, Emilie Castermans² ${ }^{2}$, Esther Korpershoek ${ }^{3}$, Irène Scagnol ${ }^{4}$, Pablo Beckers ${ }^{2}$, Vinciane Dideberg ${ }^{2}$, Vincent Rohmer ${ }^{1}$, Vincent Bours², Albert Beckers ${ }^{1}$

Affiliations

1 Department of Endocrinology, Liège Université, Liège, Belgium

2 Department of Human Genetics, Liège Université, Liège, Belgium

3 Department of Pathology, Erasmus University Medical Center, Rotterdam, The Netherlands

4 Department of Pathology, Liège Université, Liège, Belgium

Key words

familial, MAX, pancreatic neuroendocrine neoplasm, pituitary, pheochromocytoma

received 09.03.2020

accepted after revision $\quad 18.05 .2020$

published online $\quad 09.06 .2020$

Bibliography

Horm Metab Res 2020; 52: 784-787

DOI 10.1055/a-1186-0790

ISSN 0018-5043

(c) 2020. Thieme. All rights reserved.

Georg Thieme Verlag KG, Rüdigerstraße 14,

70469 Stuttgart, Germany

Correspondence

Prof. Albert Beckers MD, PhD

Chief, Department of Endocrinology Centre Hospitalier

Universitaire de Liège, Liège Université

4000 Liège

Belgium

Tel.: + 324 3667083, Fax: + 3243667261

albert.beckers@chuliege.be

\section{ABSTRACT}

Most pancreatic neuroendocrine neoplasms (pNEN) occur sporadically but they can also occur as part of multiple endocrine neoplasia type 1 (MEN1). MAX was originally described as an inherited pheochromocytoma-paraganglioma risk gene, but also has recently been implicated in pituitary tumorigenesis. Here we describe the first case of a pNEN associated with an inherited MAX gene deletion in a family with endocrine tumors. The patient was a male carrier of an intragenic exon 3 deletion inherited from his father who had recurrent pheochromocytomas and a macroprolactinoma. The patient underwent screening and hormonal studies but no pheochromocytoma-paraganglioma, pituitary or renal tumors were identified. However, abdominal magnetic resonance imaging (MRI) identified a $1 \mathrm{~cm}$ lesion in body of the pancreas. The lesion was hyperintense on T2-weighted signal, and there was hyperfixation of the tumor on 68Ga-DOTANOC PET-CT images. No biochemical evidence of pancreatic hormone excess was identified. Following a guided biopsy, a pathological diagnosis of a low grade pNEN was made and immunohistochemistry showed loss of MAX nuclear staining. Genetic analysis of the tumor tissue indicated copy number neutral loss of heterozygosity consistent with uniparental disomy. This is the first reported case of a MAX deletion associated pNEN and strengthens the argument that $M A X$ may represent an inheritable multiple endocrine neoplasia risk gene. Further analysis of germline and somatic MAX mutations/deletions in large cohorts of unexplained NEN cases could help clarify the potential role of MAX in NEN etiology.

\section{Introduction}

Pancreatic neuroendocrine neoplasms (pNEN) have an incidence of 0.48 cases per 100000 , and the frequency is rising [1]. While they are usually sporadic, pNENs can occur in the setting of multiple endocrine neoplasia type 1 (MEN1) and hence they are the subject of active surveillance in that setting [2]. Other genetic syndromes that are rarely associated with pNENs include von HippelLindau disease, neurofibromatosis type 1 (NF1), MEN4, Lynch and Cowden syndrome [3-9].
In 2011, Comino Mendez et al. identified MAX as a risk gene for the development of hereditary pheochromocytoma [10]. Germline mutations in MAX lead to the development of sporadic and familial pheochromocytoma-paragangliomas and MAX acts as a tumor suppressor gene in the MYC/MAX/MXD1 pathway [11]. While germline MAX genetic changes account for a small proportion of all known genetic forms of pheochromocytoma-paragangliomas, they appear to have an aggressive phenotype. Burnichon et al. reported that pheochromocytoma-paragangliomas patients with 
MAX mutations had an earlier age at onset as compared with non-mutated cases and MAX associated tumors are much more frequently bilateral or have multiple tumors occurring within the same gland [11]. Until recently the tumoral phenotypes associated with germline MAX mutations and rearrangements were limited to pheochromocytoma, paraganglioma and kidney neoplasms $[12,13]$. In primary tumors and cell cultures derived from small cell lung cancer, a neuroendocrine tumor, somatic MAX mutations and deletions with concurrent loss of heterozygosity $(\mathrm{LOH})$ were found to occur in $6 \%$ of cases [14]. Furthermore, two patients with gastrointestinal intestinal stromal tumors (GIST) that were negative for KIT/PDGFRA/BRAF/SDHx abnormalities (quadruple wild-type) were reported as having somatic truncating mutations in MAX [15].

An association between MAX and the development of pituitary adenomas (acromegaly or prolactinoma) has been described recently $[16,17]$. We described three cases of intragenic germline deletions in MAX that were not identified on Sanger sequencing but were established with multiplex ligation-dependent probe amplification (MLPA). Those cases had aggressive features with early onset, recurrence, bilateral pheochromocytomas or metastatic disease, in keeping with established MAX related characteristics $[11,17]$. In one kindred, the deletion was inherited by the patient's son from his father [17]. Subsequent screening of this 31-year old male, who had no medical history, was undertaken to identify tumors in known sites related to MAX mutations. Unexpectedly, abdominal imaging studies revealed a pancreatic mass, which was further investigated and characterized.

\section{Statement of Ethics}

The patient provided informed consent and the study was approved by the Ethics Committee of the CHU de Liège.

\section{Methods and Results}

As we reported previously, the patient's father had a history of recurrent pheochromocytoma and a prolactinoma in the setting of a germline intragenic exon 3 deletion in MAX [17]. The pheochromocytoma tissue had been shown to have $\mathrm{LOH}$ at the MAX locus that differed between the initial tumor and the recurrence (18 years later), indicating separate somatic "second hit" events affecting the wild-type MAX allele [17]. Family genetic studies including MLPA had identified the son as a carrier of the identical germline exon 3 MAX deletion as his father ( Fig. 1a). Screening studies were performed and included biochemical and hormonal analyses of adrenal and pituitary function, hematological, renal and liver function tests. All were normal. Abdomino-thoracic and pituitary magnetic resonance imaging (MRI) were performed and no evidence of pheochromochromocytoma/paraganglioma, pituitary adenoma, or kidney tumors was identified. On the abdominal MRI a $1 \mathrm{~cm}$ lesion in body of the pancreas was identified, which was hyperintense on T2 weighted signal ( $\mathbf{F i g}$. 1b). An ${ }^{18} \mathrm{~F}$-fluorodeoxyglucose-positron emission tomography-CT ( ${ }^{18}$ FDG-PET-CT) scan showed no enhanced uptake. There was hyperfixation of the tumor on ${ }^{68} \mathrm{Ga}$-DOTANOC PET-CT images, indicating strong $\mathrm{SST}_{2}$ expression ( $\mathbf{F i g . ~ 1 C ) . ~ N e i t h e r ~ b i o c h e m i c a l ~ e v i d e n c e ~ n o r ~ s i g n s / s y m p t o m s ~}$ of pancreatic hormone excess were identified. The patient provided informed consent and the study was approved by the Ethics Committee of the $\mathrm{CHU}$ de Liège.

To further investigate the lesion, a percutaneous ultrasound-guided fine-needle aspiration (FNA) biopsy was performed. Hematoxylin and eosin staining showed aggregations of cells with eccentric nuclei, salt and pepper chromatin pattern and a granular, eosinophilic cytoplasm ( $>$ Fig. 2a). The tissue was positive for anti-CD56, Chromogranin A and Synaptophysin and no mitoses were seen. A pathological diagnosis of a low grade pancreatic neuroendocrine tumor was made ( $\mathrm{G} 1$ grade; Ki-67: 1-2\%, mitotic index: 0 ). Immunohistochemistry of the FNA material for MAX was performed as previously described [12]; this showed neuroendocrine cells that exhibited loss of MAX nuclear staining in the setting of other normally-stained cells ( $\triangleright$ Fig. $\mathbf{2 b}$ ). Genetic analyses were also performed on the pNEN FNA tissue DNA; MLPA showed LOH and an apparent homozygous deletion of the exon 3 of MAX gene ( $\triangleright$ Fig. $\mathbf{2 c}$ ). The MLPA results and the paternal inheritance pattern strongly point copy neutral LOH involving the MAX locus due to paternal uniparental disomy (UPD) at chromosome $14 q$ as has been
I

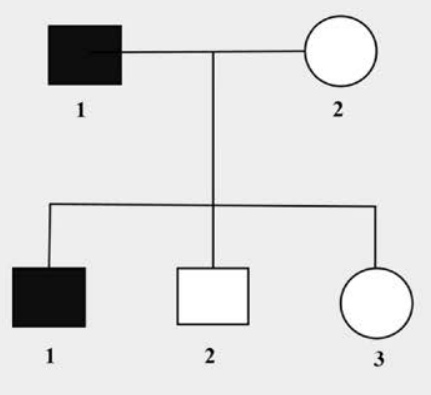

b

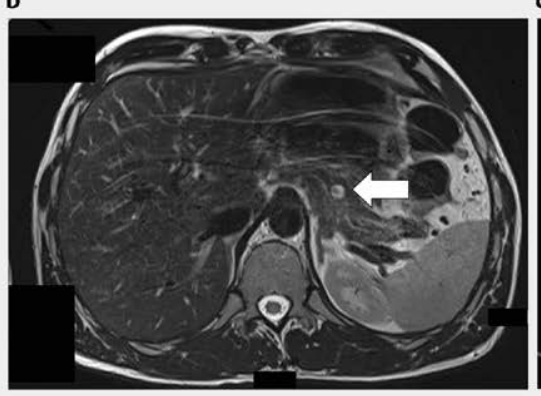

c

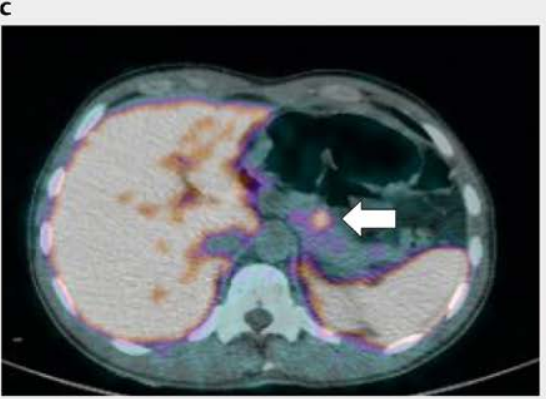

-Fig. 1 Panel a shows the genealogical tree of the family. The father (I1) had a pheochromocytoma at 32 years of age that recurred at the age of 50 and a prolactinoma that was diagnosed at the age of 49 years. His son (II1) had a pancreatic neuroendocrine tumor discovered during screening at the age of 32. Both I1 and II 1 were diagnosed with an intragenic deletion of exon 3 in MAX. Other family members were tested and had a wild-type MAX sequence and MLPA. Panel $\mathbf{b}$ shows the location of the pNEN (arrow) as a hyperintense lesion in the body of the pancreas on a T2-weighted MRI. Panel $\mathbf{c}$ shows intense uptake in the tumor (arrow) on ${ }^{68} \mathrm{Ga}$-DOTANOC PET-CT. 

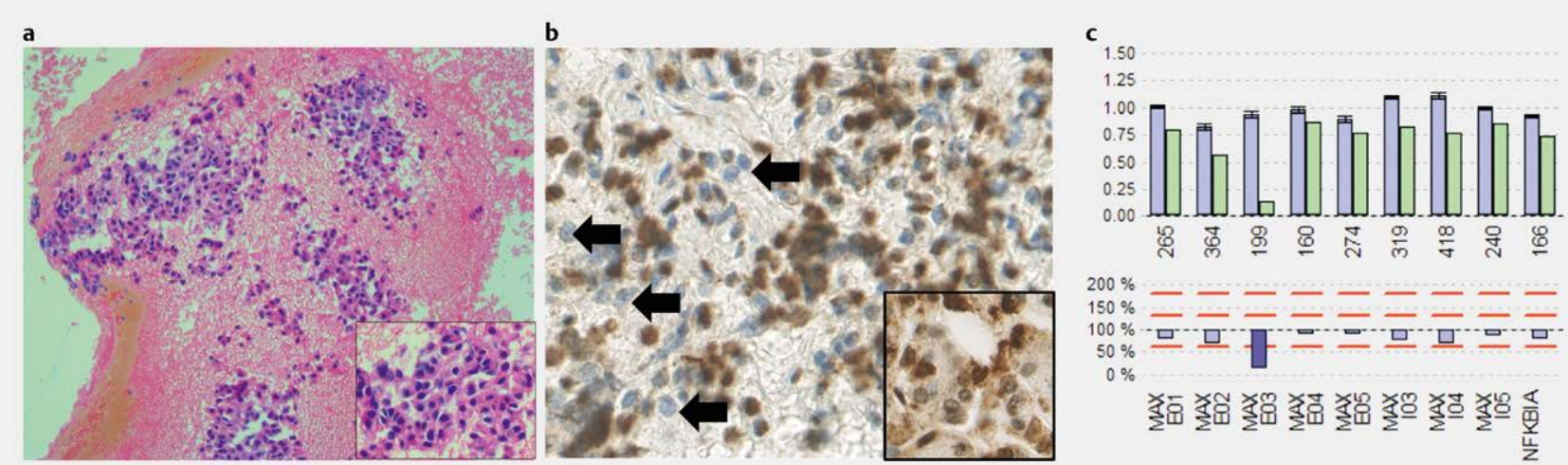

Fig. 2 Panel a shows an image of the hematoxylin and eosin stain of the tissue obtained following fine needle biopsy of the pancreatic lesion. The biopsy material shows groups of abnormal cells with generally eccentric nuclei and an eosinophilic cytoplasm. Inset is a high magnification image of a section of the tumor cells. Arrowed on Panel $\mathbf{b}$ are groups of tumoral cells that were negative for MAX staining (blue nuclei and negative cytoplasm) interspersed with groups of normal cells. Inset is an image of a positive control from a normal pancreas section demonstrating strong positive (brown) nuclear and moderately positive cytoplasmic staining. Panel c illustrates the MLPA finding on pNEN tumor DNA showing an apparent homozygous deletion in exon 3 in the MAX gene. The upper histograms show each probe coverage in the kit controls (blue) and in the patient's sample (green).

demonstrated in familial cases of MAX-related pheochromocytoma and renal oncocytoma $[11,12]$.

The patient remains under close clinical follow-up and is currently asymptomatic. On abdominal MRI at six months post-diagnosis the tumor remains stable and in light of the low grade, size $<2 \mathrm{~cm}$, low Ki-67 score, non-functional status and patient wishes, the patient is being managed with active surveillance [18].

\section{Discussion}

This is, to the best of our knowledge, the first case of a gastroenteropancreatic NEN associated with an inherited germline MAX mutation or deletion. Originally MAX mutations were described in association with pheochromocytoma, and subsequent research has further defined the clinical phenotype which can be bilateral and aggressive $[10,11,19]$. Since then MAX has been implicated in a growing number of sporadic and familial cancers, many which have a neuroendocrine origin. Emerging evidence suggests that inactivating MAX genetic abnormalities appears to lead to tumor risk at multiple endocrine and non-endocrine tissues, including pheochromocytoma, paraganglioma, renal tumors, pituitary adenomas, and GIST and SCLC $[10-17,19,20]$. Clustering of tumors within the same patient and/or kindred with MAX mutations includes pheochromocytoma-paraganglioma, pituitary adenoma, and renal tumors [10-12, 16, 17].

The past decade has seen a large volume of fundamental research on the genetics and genomics of NEN in general and pNEN in particular. The study of inherited or familial disorders provided early and important insights into pNEN pathogenesis, including sporadic disease [21]. For example, comprehensive analyses have identified mutations in genes such MEN1, VHL, TSC1, TSC2, and PTEN, which cause individual syndromic diseases, as also playing an integral role in the development of sporadic PNET [21-23]. In addition, mutations in the ATRX and DAXX genes that are involved in telomere length regulation via histone 3.3 deposition are frequently found in pNEN [2]. Subsequent work has expanded the list of recurrent genetic alterations, chromosomal loss/gain patterns and epigenetic profiles and certain pathway groupings are now evident, including, MEN1-related alterations, telomeric changes (ATRX/DAXX), abnormal cell-cycle regulation (e.g., CDKN1B), PI3K-mTORpathway disorders, and disordered chromatin remodeling or DNA and base repair dysregulation [21]. While these large-scale studies have not identified MAX mutations/deletions as a major contributor to sporadic PNEN pathogenesis, it remains to be seen if MAX intragenic copy number variations represent a contributory factor in a subgroup of cases. Taking the findings of the current study into account, it seems reasonable to suggest that surveillance of previously identified MAX carriers could be expanded to include a wider range of potential target tumors. As sporadic pheochromocytoma-paraganglioma cases without known family history can have unsuspected germline mutations in $M A X$, similar tumor risk related to MAX might be present in sporadic cases of NEN, pituitary adenoma, among others [24]. Genetic analyses of large NEN and other tumor banks should assess for intragenic deletions and complex rearrangements of $M A X$, which can be missed by some sequencing driven approaches [17].

\section{Funding Information}

This work was funded by grants from the FIRS 2018-2019, CHU de Liège, and from the JABBS Foundation, UK (to Albert Beckers).

\section{Conflict of Interest}

The authors declare that they have no conflict of interest. 


\section{References}

[1] Dasari A, Shen C, Halperin D et al. Trends in the incidence, prevalence, and survival outcomes in patients with neuroendocrine tumors in the United States. JAMA Oncol 2017; 3: 1335-1342

[2] Thakker RV, Newey PJ, Walls GV et al. Endocrine Society. Clinical practice guidelines for multiple endocrine neoplasia type 1 (MEN1). J Clin Endocrinol Metab 2012; 97: 2990-3011

[3] Di Domenico A, Wiedmer T, Marinoni I et al. Genetic and epigenetic drivers of neuroendocrine tumours (NET). Endocr Relat Cancer 2017; 24: R315-R334

[4] Hammel PR, Vilgrain V, Terris B et al. Pancreatic involvement in von Hippel-Lindau disease. The Groupe Francophone d'Etude de la Maladie de von Hippel-Lindau. Gastroenterology 2000; 119: 1087-1095

[5] Agaimy A, Vassos N, Croner RS. Gastrointestinal manifestations of neurofibromatosis type 1 (Recklinghausen's disease): Clinicopathological spectrum with pathogenetic considerations. Int J Clin Exp Pathol 2012; 5: 852-862

[6] Barrera AS, Serra Pla S, Blázquez Maña CM et al. Pancreatic non-functioning neuroendocrine tumor: A new entity genetically related to Lynch syndrome. J Gastrointest Oncol 2017; 8: E73-E79

[7] Neychev V, Sadowski SM, Zhu J et al. Neuroendocrine tumor of the pancreas as a manifestation of cowden syndrome: A case report. J Clin Endocrinol Metab 2016; 101: 353-358

[8] Mortaji P, Morris KT, Samedi V et al. Pancreatic neuroendocrine tumor in a patient with a TSC1 variant: Case report and review of the literature. Fam Cancer 2018; 17: 275-280

[9] Alrezk R, Hannah-Shmouni F, Stratakis CA. MEN4 and CDKN1B mutations: The latest of the MEN syndromes. Endocr Relat Cancer 2017; 24: T195-T208

[10] Comino-Méndez I, Gracia-Aznárez F], Schiavi F et al. Exome sequencing identifies MAX mutations as a cause of hereditary pheochromocytoma. Nat Genet 2011; 43: 663-667

[11] Burnichon N, Cascon A, Schiavi F et al. MAX mutations cause hereditary and sporadic pheochromocytoma and paraganglioma. Clin Cancer Res 2012; 18: 2828-2837

[12] Korpershoek E, Koffy D, Eussen BH et al. Complex MAX rearrangement in a family with malignant pheochromocytoma, renal oncocytoma, and erythrocytosis. J Clin Endocrinol Metab 2016; 101: 453-460
[13] Casey RT, Warren AY, Martin JE et al. Clinical and molecular features of renal and pheochromocytoma/paraganglioma tumor association syndrome (RAPTAS): Case series and literature review. J Clin Endocrinol Metab 2017; 102: 4013-4022

[14] Romero OA, Torres-Diz M, Pros E et al. MAX inactivation in small cell lung cancer disrupts MYC-SWI/SNF programs and is synthetic lethal with BRG1. Cancer Discov 2014; 4: 292-303

[15] Pantaleo MA, Urbini M, Indio V et al. Genome-wide analysis identifies MEN1 and MAX mutations and a neuroendocrine-like molecular heterogeneity in quadruple WT GIST. Mol Cancer Res 2017; 15: 553-562

[16] Roszko KL, Blouch E, Blake M et al. Case report of a prolactinoma in a patient with a novel MAX mutation and bilateral pheochromocytomas.. J Endocr Soc 2017; 1: 1401-1407

[17] Daly AF, Castermans E, Oudijk L et al. Pheochromocytomas and pituitary adenomas in three patients with MAX exon deletions. Endocr Relat Cancer 2018; 25: L37-L42

[18] Falconi M, Eriksson B, Kaltsas G et al. ENETS Consensus Guidelines update for the management of patients with functional pancreatic neuroendocrine tumors and non-functional pancreatic neuroendocrine tumors. Neuroendocrinology 2016; 103: 153-171

[19] Bausch B, Schiavi F, Ni Y et al. Clinical characterization of the pheochromocytoma and paraganglioma susceptibility Genes SDHA, TMEM127, MAX, and SDHAF2 for Gene-Informed Prevention. JAMA Oncol 2017; 3: 1204-1212

[20] Schaefer IM, Wang Y, Liang CW et al. MAX inactivation is an early event in GIST development that regulates p16 and cell proliferation. Nat Commun 2017; 8: 14674

[21] Mafficini A, Scarpa A. Genetics and epigenetics of gastroenteropancreatic neuroendocrine neoplasms. Endocr Rev 2019; 40: 506-536

[22] Scarpa A, Chang DK, Nones K et al. Whole-genome landscape of pancreatic neuroendocrine tumours. Nature 2017; 543: 65-71

[23] Jiao Y, Shi C, Edil BH et al. DAXX/ATRX, MEN1, and mTOR pathway genes are frequently altered in pancreatic neuroendocrine tumors. Science 2011; 331: 1199-1203

[24] Albattal S, Alswailem M, Moria Y et al. Mutational profile and genotype/phenotype correlation of non-familial pheochromocytoma and paraganglioma. Oncotarget 2019; 10: 5919-5931 